\title{
VARIABILIDAD ESPACIAL DE ALGUNAS PROPIEDADES QUÍMICAS EN UN ENTISOL
}

\section{SPATIAL VARIABILITY OF SOME CHEMICAL PROPERTIES IN AN ENTISOL}

\author{
Carlos Andrés Garzón Gutiérrez¹, César Andrés Cortés², Jesús Hernán Camacho-Tamayo³
}

\begin{abstract}
${ }^{1}$ Ingeniero Agrónomo, egresado de la Universidad de Cundinamarca. E-mail: granathilas@ hotmail.com; ${ }^{2}$ Ingeniero Agrícola, M.Sc. Facultad de Ing. Agronómica, Universidad de Ciencias Aplicadas y Ambientales U.D.C.A E-mail: cescortes@ udca.edu.co; ${ }^{3}$ Ingeniero Agrícola, M.Sc. Profesor Asistente. Facultad de Ingeniería, Programa de Ingeniería Agrícola, Universidad Nacional de Colombia. Cra. 45 No. 45 - 03, Bogotá, Colombia. E-mail: jhcamachot@unal.edu.co
\end{abstract}

Rev. U.D.C.A Act. E Div. Cient. 13 (1): 87-95, 2010

\section{RESUMEN}

El desarrollo y la aplicación de tecnologías para establecer el comportamiento espacial de los suelos permite optimizar el uso de los diferentes recursos agrícolas, con el fin de mejorar la sostenibilidad y la competitividad, reduciendo el riesgo de degradación ambiental y mejorando su rentabilidad. El objetivo del presente estudio fue evaluar la variabilidad espacial y la relación de algunas propiedades químicas del suelo, mediante diferentes técnicas estadísticas. El trabajo fue realizado en el municipio de Pasca (Cundinamarca), en área productora de Cyphomandra betacea (Cav.) Sendtn., en un Entisol. Se tomaron 64 muestras en una malla regular, con distancias perpendiculares entre puntos de 25 x 12,5m, a una profundidad de 0,20m, determinando carbono orgánico, $\mathrm{pH}, \mathrm{Ca}, \mathrm{Mg}, \mathrm{K}$, suma de bases y la relación Ca:Mg. Los datos fueron analizados mediante estadística descriptiva, análisis multivariado, geoestadística e interpolación por kriging. Los atributos manifestaron variabilidad baja o media, donde el $\mathrm{K}$ fue el único atributo que no presentó dependencia espacial. Se observó una fuerte relación entre los cationes, así como una estrecha relación entre el Ca y la suma de bases. Los mapas de contorno confirmaron la variabilidad espacial de las propiedades, lo que indica la conveniencia de la aplicación de insumos agrícolas mediante tasa variada. Con la adaptación de esta metodología, se puede mejorar el diseño experimental de futuras investigaciones, además de ser la base para el establecimiento de cultivos, bajo la concepción de agricultura de precisión.

Palabras clave: Geoestadística, análisis multivariado, kriging, semivariograma.

\section{SUMMARY}

Development and application of technologies for establishing the spatial behavior of soils, allow to optimize the use of different agricultural resources in order to improve the sustainability and competitiveness, reducing the risk of environmental degradation and improving its profitability. The aim of this study was to evaluate the spatial variability and the relationships of some soil chemical properties, using different statistical techniques. The reseach was carried out in the municipality of Pasca (Cundinamarca) in a Cyphomandra betacea (Cav.) Sendtn. production area, in an Entisol. On a regular grid, 64 samples were taken, with perpendicular distances between points $25 \times 12.5 \mathrm{~m}$, at $0.20 \mathrm{~m}$ of depth, evaluating organic carbon, $\mathrm{pH}, \mathrm{Ca}$, $\mathrm{Mg}, \mathrm{K}$, sum of bases and Ca:Mg relation. The data were analyzed using descriptive statistics, multivariate analysis, geostatistics and interpolation by kriging. The attributes showed low to medium variability, where the $\mathrm{K}$ was the only attribute that showed no spatial dependence. There was a strong relationship between cations, and 
a close relationship between $\mathrm{Ca}$ and the sum of bases. The contour maps confirmed the spatial variability of the properties, indicating the appropriateness of the agricultural inputs application at a variable rate. By adapting this methodology it is possible to improve the experimental design of future research, besides being the basis for the establishment of crops under precision agriculture concepts.

Key words: Geostatistics, multivariate analysis, kriging, semivariogram.

\section{INTRODUCCIÓN}

La agricultura moderna, se debe orientar a mejorar la sostenibilidad y la competitividad, reduciendo el riesgo de degradación ambiental y mejorando su rentabilidad (Molin et al. 2008). Por esto, el desarrollo y la aplicación de nuevas tecnologías, como los sistemas de posicionamiento global (GNSS), de información geográfica (SIG) y técnicas geoestadísticas, permiten identificar el comportamiento espacial de los suelos, con el fin de optimizar el uso de los diferentes recursos agrícolas y maximizar la producción de cultivos, vitales para la sostenibilidad agrícola y ambiental. Estas tecnologías permiten valorar y entender el comportamiento espacial y las diferentes relaciones entre las variables del suelo, la productividad de las plantas, así como de plagas y de enfermedades (Castrignano et al. 2002), comportamiento que ocurre a diferentes escalas, como resultado de procesos dinámicos del clima y las labores agrícolas (Molin et al. 2008).

Uno de los factores que incide acentuadamente en la dosis de fertilizantes requerido por el cultivo es la disponibilidad efectiva de nutrientes en el suelo o la capacidad que tiene el suelo para suministrar elementos nutritivos a las plantas. La evaluación de la capacidad de los suelos para suministrar nutrientes a los cultivos, se realiza, usualmente, mediante el análisis químico, para obtener información sobre cómo estan dispersos los nutrientes.

Existen diferentes herramientas estadísticas y matemáticas para identificar el comportamiento espacial de los atributos del suelo, además de su cuantificación y caracterización. Entre las técnicas más utilizadas, se encuentran la geoestadística y el interpolador kriging, herramientas útiles para la construcción de mapas y la comprensión de la variabilidad de las propiedades del suelo, en un área determinada. Estas instrumentos pueden ser aplicados a diferentes escalas en relación a datos puntuales, dependiendo de la resolución deseada en el estudio (Webster, 2008).

Los métodos geoestadísticos, a partir de muestreos sistemáticos, son adecuados para determinar la variabilidad espacial (Goovaerts, 1998), que se representa por medio de la correlación espacial, determinada por el semivariograma, siempre que el intervalo de muestreo constituye la variación en el nivel de interés (Kerry $\mathcal{E}$ Oliver, 2004). De esta forma, se verifica si una variable guarda relación entre puntos cercanos (Goovaerts, 1998), para generar información de zonas no muestreadas, mediante kriging (Vieira, 2000) y generar información sobre condiciones específicas del suelo, mediante mapas de contorno, lo cual, representa un importante principio en agricultura de precisión (Borůvka et al. 2002).

Desde el punto de vista del manejo de fertilidad de suelos y nutrición de cultivos, la implementación de esta metodología ofrece la posibilidad de realizar aplicaciones de dosis variables de fertilizantes, a partir de las condiciones del suelo y de las necesidades del cultivo, para alcanzar mayor eficiencia en la distribución de abonos. Al trabajar con dosis variables, se busca que el suelo brinde condiciones homogéneas para el desarrollo adecuado del cultivo, que repercutan en el desarrollo, en el crecimiento y en la producción.

Considerando la relevancia de las propiedades químicas del suelo en la producción de cultivos, se propuso esta investigación, con el objetivo de evaluar la variabilidad de algunas propiedades químicas y la relación existente en éstas, en un Entisol, cultivado con tomate de árbol, mediante técnicas de estadística univariada, multivariada, geoestadística y kriging.

\section{MATERIALES Y MÉTODOS}

Caracterización del área de estudio: El estudio se realizó en una área comercial de tomate de árbol (Cyphomandra betacea (Cav.) Sendtn., en la finca La Pradera, Vereda Alto del Molino, en el municipio de Pasca (Cundinamarca), ubicada en las coordenadas geográficas $4^{\circ} 18^{\prime} 38,83^{\prime \prime}$ de latitud Norte, $74^{\circ} 18^{\prime} 9,28^{\prime \prime}$ de longitud Oeste y altitud de $2058 \mathrm{~m}$. La zona presenta precipitación media anual de $1800 \mathrm{~mm}$, con periodos 
marcados entre marzo a mayo y septiembre a noviembre, temperatura media de $16^{\circ} \mathrm{C}$ y humedad relativa media de $85 \%$, con máximos mensuales de $93 \%$ y mínimos de $74 \%$. El suelo predominante a nivel de Orden para esa zona es Entisol. El paisaje es de montaña, con material parental de depósitos clásticos hidrogravigénicos, en sectores con mantos de ceniza volcánica. Previamente al establecimiento del cultivo, se realizó labranza convencional, con arado de cuchillas rotativas e incorporación de gallinaza y cal.

Muestreo y análisis de laboratorio: El muestreo del suelo, a una profundidad entre 0 y $20 \mathrm{~cm}$, a mediados del ciclo del cultivo, mediante el diseño de una malla rectangular, con distancias perpendiculares entre puntos de $25 \times 12,5 \mathrm{~m}$, tomando 64 muestras, en un área de 1,53 ha. Las propiedades determinadas para cada muestra fueron el contenido de carbono orgánico (CO), a través del método modificado de Walkley Black; $\mathrm{pH}$ medido con potenciómetro en relación suelo agua 1:1; contenidos de $\mathrm{Ca}, \mathrm{Mg}$ y $\mathrm{K}$, por medio de extracción con acetato de amonio $\mathrm{pH}$ 7,0 y lectura en equipo de absorción atómica. Con estos resultados, también se estimó la suma de bases (SB) y la relación Ca:Mg.

Análisis estadístico: Inicialmente, se llevó a cabo un análisis a través de la estadística descriptiva para todas las variables en estudio, con el fin de establecer el comportamiento, la dispersión, la tendencia y la distribución de los datos, mediante la media, mediana, coeficiente de variación, mínimo, máximo, asimetría, curtosis y la prueba de normalidad de KolmogorovSmirnov. De esta manera, se evaluó el precepto de normalidad para cada propiedad, la cual, no es indispensable, pero proporciona mejores predicciones cuando se asocia a técnicas geoestadísticas (Diggle E Ribeiro, 2000). Para el análisis del CV, se tuvo en cuenta la clasificación de Warrick E Nielsen (1980), que considera una variabilidad baja para $\mathrm{CV}$ menores del $12 \%$, variabilidad media para CV entre 12 y $60 \%$ y variabilidad alta para valores mayores a $60 \%$.

Posteriormente, se realizó el análisis multivariado, ejecutando análisis de factores por componentes principales (AFCP) y de agrupamiento jerárquico (AAJ), con el fin de identificar la relación entre las propiedades químicas. Para la construcción de estos análisis, los datos fueron previamente estandarizados, con media 0 y varianza 1 . En el AAJ, se utilizó el algoritmo Ward y la distancia euclidiana para separar un conjunto de atributos en grupos. Los resultados del AAJ, se representaron en forma gráfica (dendograma), buscando facilitar la identificación de los grupos formados por las propiedades analizadas. En el AFCP, se aplicó la rotación de Varimax. Los análisis de la estadística descriptiva y multivariada fueron practicados con el programa SPSS v. 12 (2003).

Para establecer el comportamiento espacial de las variables, se realizó el ajuste de los modelos teóricos de semivariogramas. La función del semivariograma experimental $\gamma(h)$ está definida como por:

$$
\gamma(h)=\frac{1}{2 N(h)} \sum_{i=1}^{n(h)}\left[z\left(x_{i}\right)-z\left(x_{i}+h\right)\right]^{2}
$$

siendo $Z\left(x_{i}\right)$, los valores muéstrales en los puntos $x_{i}$, en los que se tiene datos tanto en $x_{i}$ como en $x_{i}+h ; N(h)$ el número de pares de datos, separados, una distancia $h$. El semivariograma mide el promedio de la no semejanza entre los datos separados $\left(Z\left(x_{i}\right)-Z\left(x_{i}+h\right)\right)$ por un vector $h$, es decir, es calculado como la media de la diferencia del promedio cuadrado entre los componentes de las parejas de datos (Goovaerts, 1998).

Para el presente estudio, se efectuaron ajustes a modelos acotados (esférico, exponencial y gasussiano). Viera (2000) presenta una discusión respecto a las características y las condiciones que éstos deben cumplir. Estos modelos poseen tres parámetros comunes, que son el efecto pepita $\left(\mathrm{C}_{0}\right)$, la meseta $\left(\mathrm{C}_{0}+\mathrm{C}\right)$ y el rango $\mathrm{o}$ alcance (A). El efecto pepita indica la discontinuidad entre las muestras, es decir, la variabilidad espacial no detectada durante el proceso de muestreo; la meseta es el valor de la semi varianza, donde el modelo se estabiliza, exhibiendo un valor constante y el rango representa la distancia hasta donde existe correlación espacial, indicando que ya no existe correlación entre las muestras.

El modelo esférico es definido por $\gamma(\mathrm{h})=\mathrm{C}_{0}+$ $\mathrm{C}^{*}\left[1,5 *(\mathrm{~h} / \mathrm{a})-0,5^{*}(\mathrm{~h} / \mathrm{a})^{3}\right]$ para $0<\mathrm{h}<$ a y $\gamma(\mathrm{h})=\mathrm{C}_{0}$ $+\mathrm{C}$ para $\mathrm{h}>\mathrm{a}$; el modelo exponencial es definido por $\gamma(\mathrm{h})=\mathrm{C}_{0}+\mathrm{C}^{*}\left[1-\mathrm{e}^{(-3 \mathrm{~h} / \mathrm{a})}\right]$ para $0<\mathrm{h}<\mathrm{d}$, siendo $\mathrm{d}$, la máxima distancia, en la que el semivariograma esta precisado y el modelo gaussiano es definido por $\gamma(\mathrm{h})=$ $\mathrm{C}_{0}+\mathrm{C}^{*}\left[1-\mathrm{e}^{\left(-3 \mathrm{~h}^{2} / \mathrm{a}^{2}\right)}\right]$. Estos modelos fueron estimados con 
el programa GS + (Robertson, 1998), que adopta como criterios, para la selección del modelo, el mayor valor del coeficiente de determinación $\left(R^{2}\right)$, la menor suma de cuadrados de los residuos ( $\mathrm{SQR}$ ) y el valor más próximo de uno del coeficiente de correlación, obtenido por el método de validación cruzada (VC).

Una vez establecido el modelo teórico de cada propiedad, se verificó el grado de dependencia espacial (GDE), mediante la relación entre el efecto pepita y la meseta $\left(\mathrm{C} / \mathrm{C}_{0}+\mathrm{C}\right)$. El GDE es clasificado como fuerte, si es superior al $75 \%$; moderado para GDE, entre $25 \%$ y $75 \%$ y débil con GDE, inferior al 25\% (Cambardella et al. 1994). Se debe resaltar que es deseable que el efecto pepita no supere el $50 \%$ del valor de la meseta, para que el modelo de correlación espacial describa, adecuadamente, la realidad (Cressie, 1993). Cuando el GDE es próximo de cero, el modelo ajustado al semivariograma experimental, se denomina efecto pepita puro (Goovaerts, 1998) y se define por $\gamma(\mathrm{h})=\mathrm{C}_{0}$, para $\mathrm{h}>0$, denotando una distribución espacial aleatoria de la propiedad.

Una vez estimados los modelos teóricos de semivariograma, se aplicó la técnica kriging ordinario (Diggle $\varepsilon$ Ribeiro, 2000) para hacer predicción en sitios no muestreados y se construyeron mapas de contorno para cada propiedad, utilizando el programa Surfer (Golden Software Inc., 1999).

\section{RESULTADOS Y DISCUSIÓN}

Todas las propiedades analizadas presentaron una distribución simétrica, lo cual, se evidencia por lo valores próximos de la media y la mediana de cada una de las variables en estudio (Tabla 1). Esta simetría, se confirma por los valores próximos de cero de los coeficientes de asimetría y curtosis, así como por la prueba de Kolmogorov-Smirnov, la cual, fue no significativa para todas las propiedades. Este comportamiento normal de propiedades químicas es reportado por diferentes autores, en estudios realizados en diferentes tipos de suelos, bajo producción agrícola (Camacho-Tamayo et al. 2008; Outeiro et al. 2008). De acuerdo con Cressie (1993), más importante que la normalidad, es conveniente verificar que la distribución normal no presente colas muy largas para no comprometer los resultados, especialmente, cuando se realiza kriging, donde las estimaciones son basadas en valores medios (Warrick \& Nielsen, 1980). Otro hecho importante es la ocurrencia del efecto proporcional entre la media y la varianza de los datos a lo largo de una superficie, que permite estimar mesetas bien definidas, en los modelos de semivariogramas.

El catión predominante es el Ca, denotando la reciente distribución de cal en el área de estudio. Los cationes, en su conjunto, fueron los que mostraron mayor variabilidad, con valores de CV superiores al $29 \%$, con variabilidad

Tabla 1. Estadística descriptiva de $\mathrm{Ca}, \mathrm{Mg}, \mathrm{K}, \mathrm{pH}$, carbono orgánico (CO), suma de bases (SB) y relación Ca:Mg.

\begin{tabular}{|c|c|c|c|c|c|c|c|c|}
\hline Propiedad & Media & Mediana & CV, $\%$ & Mínimo & Máximo & Asimetría & Curtosis & K-S \\
\hline Ca, meq/100g & 11,04 & 10,88 & 37,99 & 3,02 & 20,41 & 0,36 & $-0,40$ & $\mathrm{~ns}$ \\
\hline Mg, meq/100g & 0,95 & 0,93 & 29,64 & 0,36 & 1,74 & 0,22 & $-0,14$ & $\mathrm{~ns}$ \\
\hline $\mathrm{K}, \mathrm{meq} / 100 \mathrm{~g}$ & 0,70 & 0,71 & 48,07 & 0,14 & 1,70 & 0,36 & 0,12 & $\mathrm{~ns}$ \\
\hline $\mathrm{pH}$ & 5,59 & 5,59 & 4,81 & 4,93 & 6,15 & $-0,15$ & $-0,41$ & $\mathrm{~ns}$ \\
\hline $\mathrm{CO}, \%$ & 6,41 & 6,40 & 11,78 & 4,20 & 8,40 & $-0,21$ & 0,58 & $\mathrm{~ns}$ \\
\hline $\mathrm{SB}, \mathrm{meq} / 100 \mathrm{~g}$ & 12,75 & 12,49 & 36,03 & 3,67 & 23,47 & 0,38 & $-0,15$ & $\mathrm{~ns}$ \\
\hline Ca:Mg & 11,21 & 11,03 & 30,54 & 5,54 & 19,14 & 0,54 & $-0,15$ & $\mathrm{~ns}$ \\
\hline
\end{tabular}

CV: coeficiente de variación; K-S: estadística del test de Kolmogorov-Smirnov $(\mathrm{p}=0,05)$. ns: no significativo 
media, comportamiento común para estos elementos en suelos, bajo producción agrícola (Carvalho et al. 2002; Oliveira et al. 2009). Por otra parte, Souza et al. (1997) afirman que los contenidos de K en el suelo es una de las propiedades más afectadas por el manejo antrópico, en términos de variabilidad. El pH registró la menor variabilidad, comportamiento que es comúnmente observado para esta propiedad, en diferentes tipos de suelos, a diferentes profundidades (Carvalho et al. 2002; Camacho-Tamayo et al. 2008; Hurtado et al. 2009). La SB y la relación Ca:Mg también manifestaron una variabilidad alta, resultados esperados, dado que estas propiedades son obtenidas a partir de los cationes.

El AAJ permitió identificar dos grupos bien definidos (Figura 1A). El primero, relacionado, principalmente, con la presencia de cationes, formado por el $\mathrm{K}, \mathrm{Mg}$, Ca y SB y el segundo grupo, se encuentra compuesto por propiedades que ayudan a describir la acidez del suelo, conformado por el CO, la relación Ca:Mg y pH. La semejanza resultante entre SB y $\mathrm{Ca}$, se debe a que
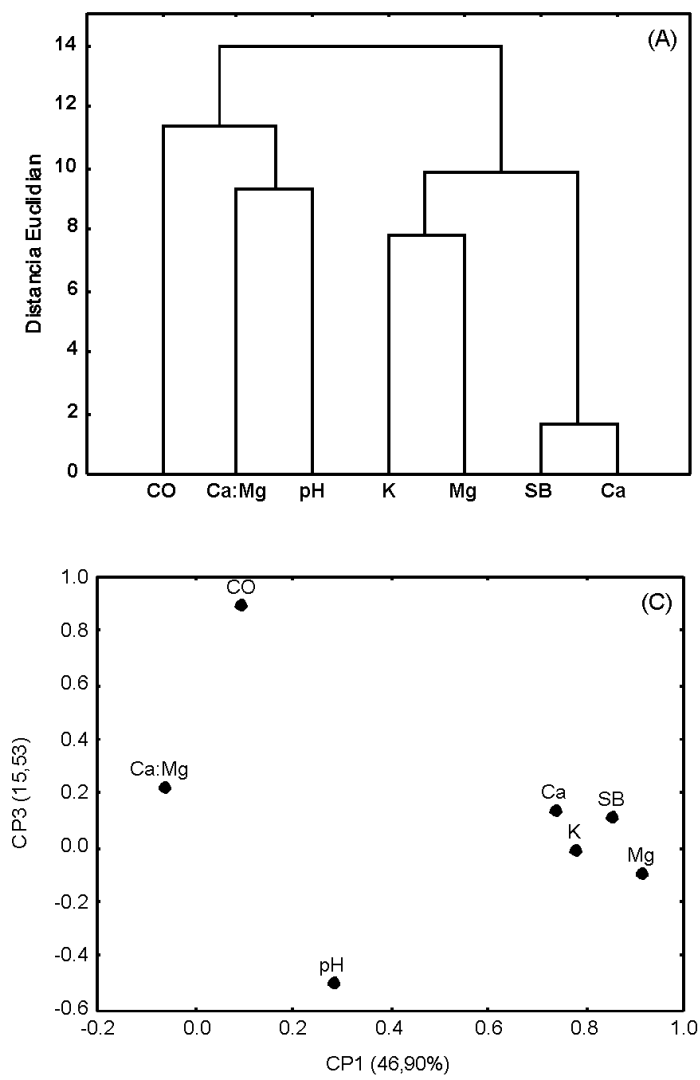

el Ca es el catión predominante y representa más del $80 \%$ del valor de SB.

En el AFCP, se observó que las propiedades formaron dos grupos definidos, concordando con los resultados obtenidos en el AAJ, cuando se analizaron los primeros dos componentes principales (Figura 1B), así como CP1 y CP3 (Figura 1C), mostrando la fuerte relación entre los cationes y $\mathrm{SB}$. El contenido de $\mathrm{CO}$, el $\mathrm{pH}$ y la relación Ca:Mg, se presentan alejados de los cationes, indicando un relación inversa.

Para el AFCP, se consideraron los tres primeros componentes, con autovalores superiores de uno, que en el presente estudio manifestaron un intervalo aceptable (Kaiser \& Rice, 1974), siendo que CP1, CP2 y CP3 explican el 84,20\% de la varianza total (Tabla 2). Se observó que los valores de la comunalidad del CO y del $\mathrm{pH}$ fueron los menores, indicando que estas propiedades son poco representativas en los componentes principales analizados.
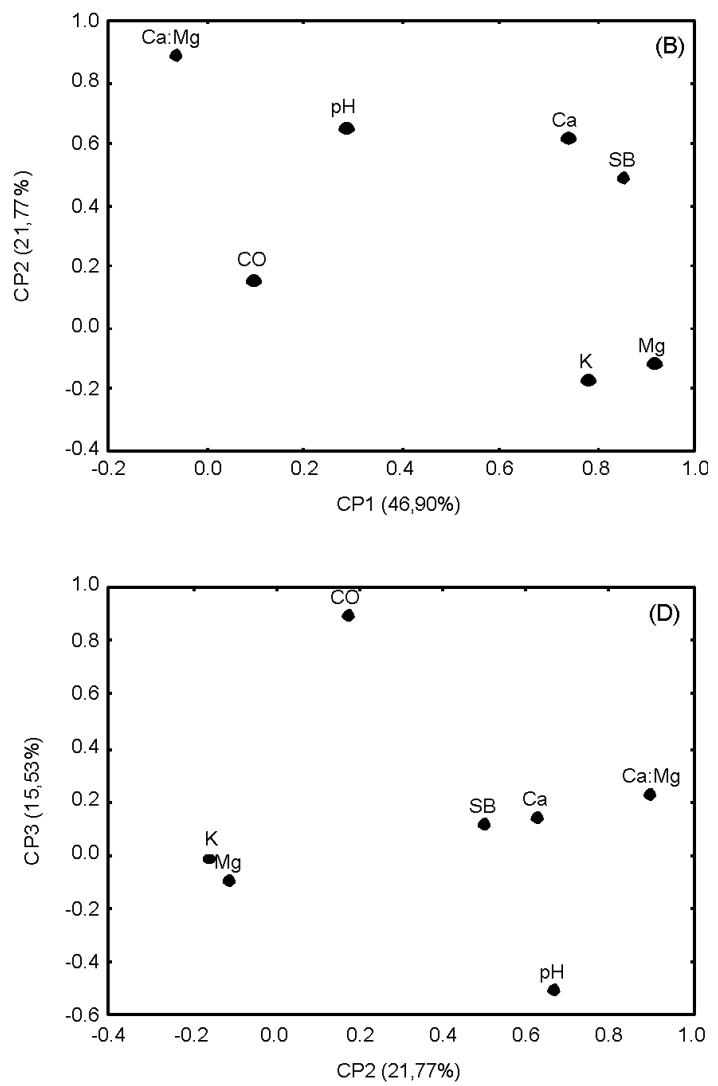

Figura 1. Dendograma resultante del análisis de agrupamiento jerárquico $(A)$ y análisis de factores de componentes principales de las propiedades químicas, mediante rotación de Varimax (B, C y D). 
Tabla 2. Coeficientes de los tres primeros componentes para $\mathrm{Ca}, \mathrm{Mg}, \mathrm{K}, \mathrm{pH}$, carbono orgánico (CO), suma de bases (SB) y relación Ca:Mg.

\begin{tabular}{|c|c|c|c|c|}
\hline Propiedad & CP1 & CP2 & CP3 & Comunalidad \\
\hline $\mathrm{Ca}$ & $\mathbf{0 , 7 3 6 7 7 2}$ & 0,625904 & 0,144349 & 1,00 \\
\hline $\mathrm{Mg}$ & $\mathbf{0 , 9 1 4 2 8 1}$ & $-0,111712$ & $-0,090013$ & 1,00 \\
\hline $\mathrm{K}$ & $\mathbf{0 , 7 7 8 1 5 4}$ & $-0,159112$ & $-0,008638$ & 1,00 \\
\hline $\mathrm{pH}$ & 0,281942 & 0,661312 & $-0,495401$ & 0,37 \\
\hline $\mathrm{CO}$ & 0,092508 & 0,169818 & $\mathbf{0 , 8 9 8 2 3 1}$ & 0,21 \\
\hline $\mathrm{SB}$ & $\mathbf{0 , 8 4 9 0 3 9}$ & 0,494831 & 0,114872 & 1,00 \\
\hline $\mathrm{Ca}: M g$ & $-0,069209$ & $\mathbf{0 , 8 9 8 5 0 5}$ & 0,231285 & 0,88 \\
\hline Autovalor & 3,28 & 1,52 & 1,09 & \\
\hline Vari. Total, \% & 46,90 & 21,77 & 15,53 & \\
\hline Var. Acum., \% & 46,90 & 68,67 & 84,20 & \\
\hline
\end{tabular}

Valores superiores de 0,7 (valor absoluto) son presentados en negrilla.

El CP1 representa el $46,90 \%$ de la varianza total, influenciado por los contenidos de $\mathrm{Ca}, \mathrm{Mg}, \mathrm{K}$ y SB, confirmando la relación directa que presentan estas propiedades. El CP2 representa 21,77\% de la varianza total, revelando una mayor influencia de la relación Ca:Mg, propiedad que está correlacionado inversamente con Mg y K, como se observa en la Figuras 1B, 1C y 1D. El CP3, con 15,53\% de la varianza total, es explicado, esencialmente, por el $\mathrm{CO}$, propiedad que mostró una correlación inversa con $\mathrm{Mg}, \mathrm{K}$ y el $\mathrm{pH}$.

Los modelos teóricos de semivariogramas isotrópicos predominantes fueron el exponencial y el esférico (Tabla 3). El K fue la única propiedad que no presentó dependencia espacial definida, es decir, la distribución espacial de K en el suelo es aleatoria, exhibiendo efecto pepita puro (EPP). El coeficiente de determinación $\left(R^{2}\right)$ siempre fue superior a 0,60 para las propiedades con dependencia espacial, siendo la relación Ca:Mg la de mejor ajuste. Estos valores del $R^{2}$, junto a los valores próximos de uno del coeficiente de validación cruzada (CVC) para todas las propiedades, indican una confiabilidad adecuada de los datos. Estudios desarrollados por diferentes autores obtuvieron ajustes a modelos teóricos de semivariogramas, para diferentes propiedades químicas (Carvalho et al. 2002; CamachoTamayo et al. 2008; Outeiro et al. 2008; Hurtado et al. 2009).
Los menores alcances se obtuvieron para el $\mathrm{pH}$, el SB y la relación Ca:Mg, con valores de 43,50, 42,20 y $64,20 \mathrm{~m}$, respectivamente, propiedades que presentaron los mayores valores del grado de dependencia espacial (GDE), clasificados como fuerte. Por otra parte, las propiedades $\mathrm{Ca}, \mathrm{Mg}$ y $\mathrm{CO}$ mostraron los mayores alcances, con GDE moderados, teniendo en común que estas propiedades registraron también los menores valores del $\mathrm{R}^{2}$, es decir, para el presente estudio, se observó que un mayor alcance corresponde, razonablemente, con un menor $\mathrm{R}^{2} \mathrm{y}$ un menor GDE.

Los mapas de contorno confirman la variabilidad espacial de las propiedades (Figura 2), lo que indica, la conveniencia de la aplicación de insumos agrícolas, mediante técnicas de tasa variada, de acuerdo a los requerimientos del cultivo, para mejorar su uso, buscando disminuir costos de producción y el impacto ambiental, debido a excesos o deficiencias que se pueden presentar en diferentes zonas, en la distribución de fertilizantes o correctivos, cuando se realiza una distribución homogénea (Molin et al. 2008).

Estos mapas también corroboran la relación existente entre las diferentes propiedades, como se encontró en el AAJ y en el AFCP. La incorporación de cal en la zona de estudio influyó en la semejanza de los mapas de 
Tabla 3. Parámetros de los modelos ajustados de semivariogramas para $\mathrm{Ca}, \mathrm{Mg}, \mathrm{K}, \mathrm{pH}$, carbono orgánico (CO), suma de bases (SB) y relación Ca:Mg.

\begin{tabular}{|c|c|c|c|c|c|c|c|}
\hline Propiedad & Modelo & $\mathrm{Co}$ & $\mathrm{Co}+\mathrm{C}$ & $\mathrm{A}, \mathrm{m}$ & $\mathrm{C} / \mathrm{Co}+\mathrm{C}$ & $\mathrm{R}^{2}$ & $\mathrm{CVC}$ \\
\hline $\mathrm{Ca}$ & Exponencial & 4,76 & 15,36 & 78,30 & 0,69 & 0,65 & 0,87 \\
\hline $\mathrm{Mg}$ & Esférico & 0,05 & 0,14 & 112,00 & 0,64 & 0,64 & 0,88 \\
\hline $\mathrm{K}$ & $\mathrm{EPP}$ & 0,09 & 0,09 & & & & \\
\hline $\mathrm{pH}$ & Exponencial & 0,01 & 0,05 & 43,50 & 0,90 & 0,73 & 0,98 \\
\hline $\mathrm{CO}$ & Esférico & 0,17 & 0,41 & 102,40 & 0,59 & 0,64 & 0,89 \\
\hline $\mathrm{SB}$ & Esférico & 3,04 & 24,09 & 42,20 & 0,87 & 0,70 & 0,87 \\
\hline $\mathrm{Ca}: \mathrm{Mg}$ & Exponencial & 1,31 & 8,92 & 64,20 & 0,85 & 0,82 & 0,88 \\
\hline
\end{tabular}

EPP: Efecto pepita puro; CVC: coeficiente da validación cruzada
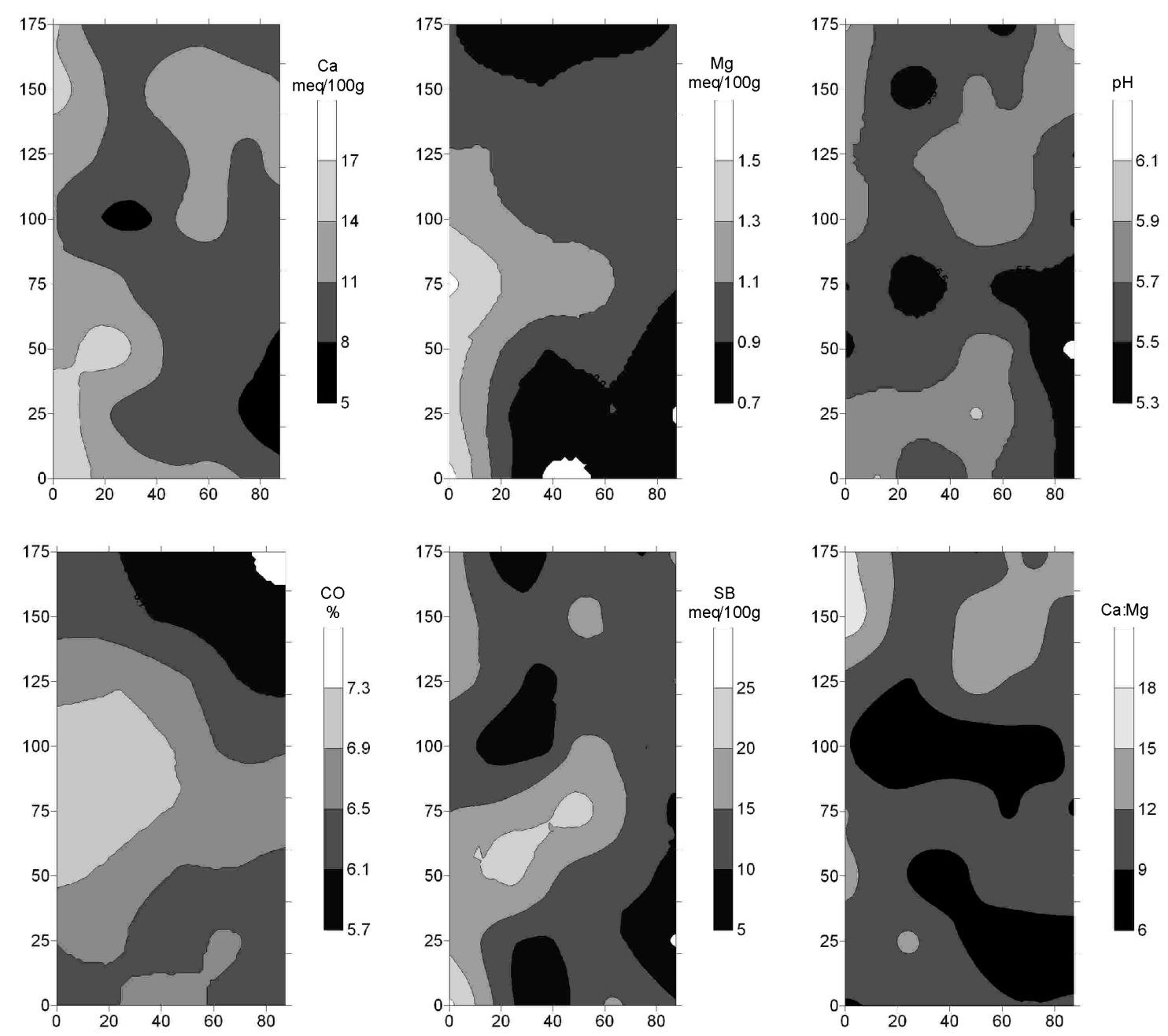

Figura 2. Mapas de contorno obtenidos mediante kriging para $\mathrm{Ca}, \mathrm{Mg}, \mathrm{K}, \mathrm{pH}$, carbono orgánico (CO), suma de bases (SB) y relación Ca:Mg. 
contorno de $\mathrm{Ca}$ y $\mathrm{Mg}$, donde zonas de alto contenido de Ca corresponden, razonablemente, a zonas de alto contenido de $\mathrm{Mg}$ y viceversa. También, se confirma la influencia del Ca en la SB, por la similitud de los mapas de estas propiedades. El mapa de contorno del CO no permitió identificar relaciones espaciales definidas con los mapas obtenidos para las otras propiedades.

Estos resultados, analizados mediante diferentes técnicas estadísticas, permitieron identificar y caracterizar el comportamiento de las diferentes propiedades del suelo, así como las relaciones existentes entre ellas. Mediante la aplicación de esta metodología, se puede mejorar el diseño experimental de futuras investigaciones, además de ser la base para el establecimiento de cultivos, bajo la concepción de agricultura de precisión, dado que permiten identificar zonas de manejo y establecer redes de muestreo, que disminuyan los costos de los análisis de suelos.

Conflictos de interés: La investigación y el manuscrito se realizaron con la colaboración de todos los autores, quienes declaramos que no existe ningún conflicto de intereses que ponga en riesgo la validez de los resultados presentados.

\section{BIBLIOGRAFÍA}

1. BORŮVKA, L., DONÁTOVÁ, H.; NĚMEČEK, K.. 2002. Spatial distribution and correlation of soil properties in a field: a case study. Rostlinná Výroba. 48(10):425-432.

2. CAMACHO-TAMAYO, J.H.; LUENGAS, C.A.; LEIVA. F.R. 2008. Effect of agricultural intervention on the spatial variability of some chemical properties of soils in the Eastern Planes of Colombia. Chilean J. Agr. Res. 68(1):42-55.

3. CAMBARDELLA, C.A.; MOORMAN, T.B.; NOVAK, J.M.; PARKIN, T.B.; KARLEN, D.L.; TURCO, R.F.; KONOPKA, A.E. 1994. Field-scale variability of soil properties in Central Iowa Soils. Soil Sci. Soc. Am. J. 58(5):1501-1511.

4. CARVALHO, J.R.P. de; SILVEIRA, P.M. da; VIEIRA, S.R. 2002. Geoestatística na determinação da variabilidade espacial de características químicas do solo sob diferentes preparos. Pesquisa Agropec. Brás. 37(8):1151-1159.

5. CASTRIGNANO, A.; MAIORANA, M.; FORNARO, F.; LOPEZ, N. 2002. 3D spatial variability of soil strength and its change over time in a durum wheat field in Southern Italy. Soil \& Tillage Res. 65(1):95-108.

6. CRESSIE, N. 1993. Statistics for spatial data, John Wiley E Sons, New York. 928p.

7. DIGGLE, P.J.; RIBEIRO, J.R. 2000. Model Based Geostatistics. $1^{a}$ ed. São Paulo: Associação Brasileira de Estatística. 129p.

8. GOLDEN SOFTWARE. 1999. Surface mapping system Inc. Surfer. Surfer version 7.00. Golden Software, Inc. Colorado. 619p.

9. GOOVAERTS, P. 1998. Geostatistical tools for characterizing the spatial variability of microbiological and physico-chemical soil properties. Biol. Fert. Soils. 27(4):315-334.

10. HURTADO, S.M.C.; SILVA, C.A.; RESENDE, A.V. DE; VON PINHO, R.G.; INÁCIO, E.S.B.; HIGASHIKAWA, F.S. 2009. Spatial variability of soil acidity attributes and the spatialization of liming requirement for corn. Ciência e Agrotecn. 33(5):13511359.

11. KAISER, H.F.; RICE, J. 1974. Little Jiffy Mark IV. Educ. Psychol. Measurement. 34(1):111-117.

12. KERRY, R.; OLIVER, M. 2004. Average variograms to guide soil sampling. International J. Appl. Earth Obs. Geoinform. 5(4):307-325.

13. MOLIN, J.P.; LEIVA, F.R.; CAMACHO-TAMAYO, J.H. 2008. Tecnología de la agricultura de precisión en el contexto de la sostenibilidad. En: Leiva, F.R. Ed.. Agricultura de precisión en cultivos transitorios. Bogotá, Universidad Nacional de Colombia. p.13-41.

14. OLIVEIRA, P.C.G.; FARIAS, P.R.S.; LIMA, H.V.; FERNANDES, A.R.; OLIVEIRA, F.A.; PITA, J.D. 2009. Variabilidade espacial de propriedades químicas 
do solo e da produtividade de citros na Amazônia Oriental. Rev. Brás. Engenharia Agr. Amb. 13(6):708-715.

15. OUTEIRO, L.; ASPERÓ, F.; ÚBEDA, X. 2008. Geostatistical methods to study spatial variability of soil cations after a prescribed fire and rainfall. Catena. 74(3):310-320.

16. ROBERTSON, G.P. 1998. GS+ geostatistics for the environmental sciences: GS+ user's guide. Plainwell: Gamma Design Software. 152p.

17. SPSS Inc. 2003. SPSS statistical software. Version 12.0. Illinois.

18. SOUZA, L.S.; COGO, N.; VIEIRA, S.R. 1997. Variabilidade de propriedades físicas e químicas do solo em um pomar cítrico. Rev. Brás. Ciencia do Solo. 21(3):367-372.
19. VIEIRA, S.R. 2000. Geoestatística em estudos de variabilidade espacial do solo. In: Novais, P.F.; Álvarez, V.H.; Schaefer, C.E.G.R eds. Tópicos em ciência do solo. Viçosa: Soc. Bras. Ciência Solo. $1: 1-54$.

20. WARRICK, A.W.; NIELSEN, D.R. 1980. Spatial variability of soil physical properties in the field. En: Hillel, D. (Ed). Applications of soil physics. New York: Academic Press. p.319-344.

21. WEBSTER, R. 2008. Soil science and geostatistics. In: Krasilnikov, P.; Carrey, F.; L. Montanarella, L. eds. Soil geography and geostatistics - concepts and applications. European Commission, Joint Research Centre, Institute for Environment and Sustainability. p.1-11.

Recibido: Noviembre 3 de 2009

Aceptado: Marzo 11de 2010 